\title{
Provision of asthma medications in developing countries: a view from the pharmaceutical industry
}

\author{
R W Fuller
}

Watson and Lewis, in their survey of expatriate doctors in Africa and Asia published in this issue of Thorax, ${ }^{1}$ highlight the relatively low use of inhaled steroids in the treatment of asthma throughout those regions. Glaxo Wellcome and other manufacturers market both salbutamol and beclomethasone in aerosol and powder form in most countries in Africa and Asia. The relatively low use of inhaled beclomethasone is consistent with our own internal market research.

There are two reasons why, in particular, inhaled steroids are not more universally available. Firstly, the infrastructure within a country may not favour adequate distribution of pharmaceuticals to some more remote areas. These logistic difficulties, complicated in some countries by civil unrest, may prohibit the availability of pharmaceuticals on a wide scale.

The second and perhaps more important reason, as highlighted by Watson and Lewis, is that the metered dose inhaler beclomethasone dipropionate, even sold at generic prices, can still account on a unit basis for approximately $20 \%$ of a monthly salary in some countries. Unlike tablets which can be split from bulk, it is not possible to do this for a metered dose aerosol and so reduce the initial outlay for the patients. It is unlikely that there is much scope for reduction of the unit cost of beclomethasone dipropionate inhalers as these are currently sold at generic prices. It should be noted that the final selling price is not only in the hands of the manufacturer as customs duty, tax, and distribution costs may account for a high proportion of the price to the patient. However, there is an increasing awareness that the prescription of unit dose powder systems such as Rotacaps or Rotadisks allows a reduction in the outlay required by the patient into manageable payments. Indeed, Glaxo Wellcome is continuing to look at potential developments of unit dose systems to make a fuller range of inhaled drugs available to this patient population.

Notwithstanding the political and economic issues around the use of inhaled steroids in the treatment of asthma, it should also be noted that the use of inhaled medication in a number of African and Asian countries does not have the same tradition as in Western Europe and this also contributes to a higher use of oral medication. In developing countries with limited resources asthma is not a priority chronic medical condition due to a lack of data on the prevalence of the disease and a limited knowledge of the disease.

Prevalence data are now becoming available with well publicised prevalence trials such as the ISAAC study which has gathered data on the 6-7 and 13-14 year age groups in a large number of countries. As these and other data become more widely available they will be used by healthcare professionals to support the prioritisation of asthma as a chronic disease and establish it on the healthcare agenda. If this is achieved, then a commitment to funding education and medication is likely to follow.

The publication of the GINA guidelines in 1992, developed in collaboration with the WHO, has stimulated knowledge about the disease and has helped to bring it onto the agenda in many developing countries. These guidelines extensively review all aspects of the disease and are seen as a framework for asthma management and, moreover, give the opportunity for local interpretation in terms of treatment regimens which mirror drug availability. Continued education and promotion of international guidelines will lead to an increase in the use of inhaled medication in the developing countries. It is therefore important that the availability of inhaled steroids does not inhibit their increasing acceptance in the treatment of asthma in the developing world.

Director, Respiratory Medicine,

R W FULLER

Respiratory, International Medical Affairs,

Glaxo Research \& Development Ltd,

Stockley Park West,

Uxbridge,

Middlesex UB11 1BT, UK

1 Watson JP, Lewis RA. Is asthma treatment affordable in developing countries? Thorax 1997;52:605-7. 